PROCEEDINGS OF THE

AMERICAN MATHEMATICAL SOCIETY

Volume 134, Number 4, Pages 949-957

S 0002-9939(05)08048-2

Article electronically published on July 25, 2005

\title{
RATIONAL FUNCTIONS AND REAL SCHUBERT CALCULUS
}

\author{
A. EREMENKO, A. GABRIELOV, M. SHAPIRO, AND A. VAINSHTEIN
}

(Communicated by John R. Stembridge)

\begin{abstract}
We single out some problems of Schubert calculus of subspaces of codimension 2 that have the property that all their solutions are real whenever the data are real. Our arguments explore the connection between subspaces of codimension 2 and rational functions of one variable.
\end{abstract}

\section{INTRODUCTION}

Let $\left\{W_{j}\right\}_{j=1}^{q}$ be a finite collection of projective subspaces in general position in the complex projective space $\mathbf{P}^{d}, a_{j}=\operatorname{dim} W_{j}$, and

$$
1 \leq a_{j} \leq d-1, \quad \sum_{j=1}^{q} a_{j}=2 d-2 .
$$

We consider the following

Problem 1. Enumerate subspaces $X \subset \mathbf{P}^{d}$ of codimension 2 which intersect each $W_{j}$ non-transversally, that is,

$$
\operatorname{dim} X \cap W_{j} \geq a_{j}-1 \quad \text { for every } j \in[1, q] .
$$

If the given subspaces $W_{j}$ are in general position, then the number of such complex subspaces $X$ is finite and can be obtained by using Pieri's formula from Schubert calculus [3, Ch. I, sect. 5].

This number turns out to be the Kostka number corresponding to the shape $2 \times(d-1)$ whose definition we recall. Let $\boldsymbol{a}=\left(a_{1}, \ldots, a_{q}\right)$. Consider the Young diagrams of the shape $2 \times(d-1)$. They consist of two rows of length $d-1$. A semistandard Young tableau SSYT of shape $2 \times(d-1)$ is a filling of such a diagram by positive integers, such that an integer $k$ appears $a_{k}$ times, the entries are strictly increasing in the columns and non-decreasing in the rows. The corresponding Kostka number $K_{\boldsymbol{a}}$ is the number of such SSYT. Schubert calculus interpretation of these numbers shows that $K_{\boldsymbol{a}}$ does not change if we permute the coordinates of $\boldsymbol{a}$. For a purely combinatorial proof of this see [5, Cor. 1.2.9] or [10, Theorem 7.10.2].

In this paper we treat Problem 1 over the field of real numbers. A subspace $X \subset \mathbf{P}^{d}$ is called real if it can be defined by equations with real coefficients, or, equivalently, if $X$ is generated by a set of real points. It follows from a general

Received by the editors August 25, 2004 and, in revised form, October 29, 2004.

2000 Mathematics Subject Classification. Primary 14P05; Secondary 26C15.

The authors were supported by NSF grants DMS-0100512 and DMS-0244421 (A.E.), DMS0200861 and DMS-0245628 (A.G.), and DMS-0401178 (M.S.); and by the BSF grant 2002375 (M.S. and A.V.) and by the Institute of Quantum Science, MSU (M.S.).

(C)2005 American Mathematical Society Reverts to public domain 28 years from publication 
result of Sottile [7] that there exist configurations of real subspaces $W_{j}$ such that all solutions $X$ of Problem 1 are real. The question is to find an explicit condition on $W_{j}$ which would imply that all solutions are real.

The following condition was proposed by B. and M. Shapiro (see [8, 9]). Let $E: \mathbf{P}^{1} \rightarrow \mathbf{P}^{d}$ denote the rational normal curve; in homogeneous coordinates $E(z)=\left(1: z: \ldots: z^{d}\right)$. The B. and M. Shapiro conjecture for the case of subspaces of codimension 2 says that if all $W_{j}$ are osculating $E$ at distinct real points, then all solutions $X$ of Problem 1 are real. This conjecture was proved in [1. B. and M. Shapiro made a similar conjecture for enumerative problems involving subspaces of arbitrary codimension but in this paper we only consider the case of codimension 2 .

In this paper we consider an extension of the result in [1] to the case that $W_{j}$ are spanned by some finite sets of real points on $E$.

Let $\left\{A_{j}\right\}_{j=1}^{q}$ be a collection of finite sets on the circle $\mathbf{R} \mathbf{P}^{1} \subset \mathbf{P}^{1}$. We say that this collection is separated if there exist pairwise disjoint closed $\operatorname{arcs} I_{j} \subset \mathbf{R} \mathbf{P}^{1}$ such that $A_{j} \subset I_{j}$ for $1 \leq j \leq q$. Our main result is

Theorem 1. Let $\left\{A_{j}\right\}_{j=1}^{q}$ be a separated collection of finite sets in $\mathbf{R P}^{1}$, each set $A_{j}$ containing $a_{j}+1$ points, such that (1) holds, and let $W_{j}$ be subspaces in $\mathbf{P}^{d}$ spanned by the sets $E\left(A_{j}\right)$. Then all subspaces $X$ of codimension 2 which satisfy (2) are real. For a generic configuration of $A_{j}$ there are exactly $K_{\boldsymbol{a}}$ such subspaces $X$.

In general, the condition that $A_{j}$ are separated cannot be removed. Let us consider several special cases.

1. Let $q=2, a_{1}=a_{2}=d-1$. Then the problem always has one solution, this solution is real, and the condition that $A_{j}$ are separated is redundant.

2. Let us consider the limiting situation when all points in each $A_{j}$ collide. Here $a_{j}=$ card $A_{j}-1$ are arbitrary integers satisfying (11). Then $W_{j}$ are subspaces osculating $E$ at some real points; this case was considered in [1, 2]. We will show that this situation is generic enough and the number of solutions is exactly $K_{\boldsymbol{a}}$, for any choice of the real points (Theorem 3 below). If $\boldsymbol{a}=(1, \ldots, 1)$, then $K_{\boldsymbol{a}}$ is the Catalan number

$$
K_{(1, \ldots, 1)}=\frac{1}{d}\left(\begin{array}{c}
2 d-2 \\
d-1
\end{array}\right) .
$$

3. Now we consider the case that $a_{1} \in[1, d-1]$ is arbitrary and all the rest of $a_{j}$ are equal to 1 . The Kostka number in this case is

$$
K_{\left(a_{1}, 1, \ldots, 1\right)}=\frac{a_{1}+1}{d}\left(\begin{array}{c}
2 d-2-a_{1} \\
d-1
\end{array}\right) .
$$

In the case that the two points of each $A_{j}, j \geq 2$ collide, Problem 1 is equivalent to the following problem of enumeration of flags.

Consider flags $F=\left(F_{1}, F_{2}\right)$ in $\mathbf{P}^{d}$, where

$$
F_{2} \subset F_{1} \text { and } \operatorname{codim} F_{i}=i, \quad i=1,2 .
$$

Suppose that $A_{1}=\left\{x_{0}, \ldots, x_{a_{1}}\right\} \subset \mathbf{R P}^{1}$ and $A_{j}=\left\{y_{j}^{(2)}\right\}$, where the superscript 2 indicates that each point $y_{j}$ is taken with multiplicity $2, j=2, \ldots, 2 d-2-a_{1}$. Our condition on the flags $F$ is that $F_{1}$ contains the points $E\left(x_{k}\right), 0 \leq k \leq a_{1}$, and 
$F_{2}$ intersects the tangent lines $W_{j}$ to $E$ at $y_{j}, j=2, \ldots, 2 d-2-a_{1}$. We want to enumerate such flags $F$.

The condition on $F_{1}$ implies that $F_{1}$ contains $W_{1}$, the subspace spanned by $E\left(x_{k}\right), 0 \leq k \leq a_{1}$. If $x_{k}$ are in general position, then $\operatorname{dim} W_{1}=a_{1}$. Now $W_{1} \subset F_{1}$ and $F_{2} \subset F_{1}$ imply that

$$
\operatorname{dim} W_{1} \cap F_{2} \geq a_{1}-1,
$$

and the remaining conditions on $F_{2}$ are

$$
\operatorname{dim} F_{2} \cap W_{j} \geq a_{j}-1=0, \quad j=2, \ldots, 2 d-2-a_{1} .
$$

In the opposite direction, suppose that $F_{2}$ satisfies (5) and (6), and define $F_{1}$ as the span of $F_{2}$ and $W_{1}$. Then codim $F_{1}=1$ and $F_{2} \subset F_{1}$.

Thus our problem of flag enumeration is equivalent to the problem of enumeration of subspaces $F_{2}$ of codimension 2 satisfying (5) and (6), which is a special limiting case of Problem 1. Assuming that the sets $A_{j}$ are separated, Theorem 1 implies that all solutions are real, and for generic choice of points $x_{i}$ and $y_{j}$ the number of these solutions is given by (4). This problem of enumeration of flags was subject to extensive computer experiments [6].

In these experiments the role of the separation condition was discovered: when it holds, all solutions are real; while when it does not hold, many configurations of points $A_{j}$ give fewer real solutions than the upper estimate from Schubert calculus.

All of the authors thank Frank Sottile for stimulating discussions and MSRI for the opportunity to work together during the semester "Topological methods in real algebraic geometry" in Spring 2004. M. S. and A. V. thank MPIM, Bonn, for their hospitality, where the final version of the paper was written. We also thank Slava Kharlamov and the referee for their valuable comments.

\section{RATIONAL FUNCTIONS}

The method of this paper is based on the relation between subspaces of codimension 2 and rational functions of one variable.

Let $G=G(d-1, d+1)$ be the Grassmannian of projective subspaces of dimension $d-2$ in $\mathbf{P}^{d}$. Every such subspace $X$ can be defined by two equations in homogeneous coordinates

$$
\begin{aligned}
& b_{0,0} z_{0}+\ldots+b_{0, d} z_{d}=0 \\
& b_{1,0} z_{0}+\ldots+b_{1, d} z_{d}=0 .
\end{aligned}
$$

We put into correspondence to this system a rational function

$$
f(z)=\frac{b_{0,0}+b_{0,1} z+\ldots+b_{0, d} z^{d}}{b_{1,0}+b_{1,1} z+\ldots+b_{1, d} z^{d}}
$$

Choosing another system of equations of the form (7) that defines the same $X$ results in replacing $f$ by $\phi \circ f$, where $\phi$ is a rational function of degree 1 . We will call rational functions $f_{1}$ and $f_{2}$ equivalent if $f_{1}=\phi \circ f_{2}$, $\operatorname{deg} \phi=1$. Let $D \subset G$ be the subset that corresponds to reducible rational functions (those whose numerator and denominator have a non-constant common factor). Then $G \backslash D$ is in bijective correspondence with equivalence classes of non-constant rational functions of degree at most $d$.

A rational function is called real if it maps $\mathbf{R} \mathbf{P}^{1}$ into itself. An equivalence class is called real if it contains a real rational function. Real classes correspond to the real elements of $G$. 
Now we translate conditions (2) to the language of rational functions. Let $W$ be the subspace of dimension $a$ spanned by the points $E\left(z_{k}\right), 0 \leq k \leq a$, on the rational normal curve. The condition that $X$ given by (7) intersects $W$ non-transversally means that

$$
\operatorname{rank} B C=1
$$

where

$$
B=\left(\begin{array}{ccc}
b_{0,0} & \ldots & b_{0, d} \\
b_{1,0} & \ldots & b_{1, d}
\end{array}\right),
$$

and $C$ is the matrix whose columns are $\left(1, z_{k}, \ldots, z_{k}^{d}\right), 0 \leq k \leq a$. Equation (9) says that the two rows of $B C$ are proportional, which is equivalent to

$$
f\left(z_{0}\right)=f\left(z_{1}\right)=\ldots=f\left(z_{a}\right) .
$$

Thus in the case that each $W_{j}$ is the span of $a_{j}+1$ points on the rational normal curve, Problem 1 is equivalent to the following interpolation-type problem for rational functions.

Problem 1'. For given subsets $A_{j} \subset \mathbf{P}^{1}$, of cardinality $a_{j}+1$, where $a_{j}$ satisfy (11), enumerate classes of rational functions $f$ of degree $d$ with the property that for every $j, f$ is constant on $A_{j}$.

Thus our Theorem 1 is equivalent to

Theorem $\mathbf{1}^{\prime}$. If the sets $A_{j} \subset \mathbf{R P}^{1}$ are separated, then all solutions of Problem $\mathbf{1}^{\prime}$ are real. For generic subsets $A_{j} \subset \mathbf{R P}^{1}$ the number of classes of real rational functions solving Problem $1^{\prime}$ is $K_{\boldsymbol{a}}$.

It is not known whether the genericity condition in the second statement of Theorem $1^{\prime}$ can be removed. It can be removed in the following special case.

Theorem 2. Let $A_{1}, \ldots, A_{q}$ be separated sets on the real line, card $A_{j}=a_{j}+1 \in$ $[2, d]$, and

$$
\sum_{j=1}^{q} a_{j}=d-1 .
$$

Then there exists a unique class of polynomials $p$ of degree d, satisfying the conditions that $p$ is constant on $A_{j}$ for $1 \leq j \leq q$.

Proof. Let $A_{j}=\left\{z_{j, 0} \ldots z_{j, a_{j}}\right\}$, where

$$
z_{j, k}<z_{j, m}, \quad \text { for } \quad 0 \leq k<m \leq a_{j}, 1 \leq j \leq q \text {, }
$$

and

$$
z_{j_{1}, k}<z_{j_{2}, m} \text { for } 1 \leq j_{1}<j_{2} \leq q \text { and all } m, k \text {. }
$$

Every polynomial of degree at most $d$ is equivalent to a unique polynomial of the form

$$
p(z)=z^{n}+\ldots+b_{1} z, \quad \text { where } n \leq d .
$$

Conditions of the theorem mean that

$$
p\left(z_{j, k}\right)=p\left(z_{j, m}\right), \quad \text { for } \quad 0 \leq k<m \leq a_{j}, 1 \leq j \leq q .
$$


This is a system of linear equations with respect to coefficients of $p$, and it is easy to see that the coefficients of this system are real. Thus the system (15) has a unique solution of the form (14) if and only if it has a unique real solution of this form.

Now suppose that our system (15) has two solutions of the form (14). Subtracting these solutions we obtain another solution which is a real polynomial $p_{0}$ of degree strictly less than $d$, and

$$
p_{0}(0)=0 \text {. }
$$

On the other hand, Rolle's theorem, (12) and (15) imply that the derivative $p_{0}^{\prime}$ has at least $a_{j}$ zeros on each interval $\left[z_{j, 0}, z_{j, a_{j}}\right]$ for $1 \leq j \leq q$ and, as these intervals are disjoint by (13), the total number of zeros of this derivative is at least $\sum a_{j}=d-1$. So $p_{0}^{\prime}=0$ and we conclude from (16) that $p_{0}=0$. This proves the theorem.

In the limit, when in each group $A_{j}$ all points collide to one point $x_{j}$, we recover from Theorem $1^{\prime}$ the main result of [1]: all rational functions whose critical points are real are equivalent to real rational functions.

In this degenerate case we have an important additional information:

Theorem 3. The number of classes of rational functions of degree $d$ with arbitrarily prescribed real critical points of given multiplicity is exactly $K_{\boldsymbol{a}}$, for any choice of the real critical points, where $\boldsymbol{a}=\left(a_{1}, \ldots, a_{q}\right)$ is the vector of multiplicities.

Our proofs of Theorems $1^{\prime}$ and 3 are based on the results in [1, 2] which we recall in the next section.

\section{Proof of Theorems $1^{\prime}$ And 3}

It is sometimes convenient to replace $\mathbf{R} \mathbf{P}^{1}$ by the unit circle $\mathbf{T}$ as in 1 . We always assume that $\mathbf{T}$ is equipped with the counterclockwise orientation. We denote by $\mathbf{s}: \mathbf{P}^{1} \rightarrow \mathbf{P}^{1}$ the reflection with respect to $\mathbf{T}$, that is $\mathbf{s}(z)=1 / \bar{z}$.

We fix $d \geq 2$. Let $R$ be the class of all rational functions $f$ such that $f(\mathbf{T}) \subset \mathbf{T}$ and all critical points of $f$ are simple and belong to $\mathbf{T}$. We equip $R$ with the following topology (see [2]): a sequence $\left(f_{k}\right)$ converges to $f$ if there exists a finite set $S \subset \mathbf{P}^{1}$ such that $f_{k} \rightarrow f$ uniformly on compact subsets of $\mathbf{P}^{1} \backslash S$.

First we recall a parametrization of the class $R$. Let $f$ be a function of the class $R$, and $v_{0}$ a critical point of $f$. The full preimage $\Gamma=f^{-1}(\mathbf{T})$ defines a cell decomposition of $\mathbf{P}^{1}$. We call the pair $\gamma=\left(\Gamma, v_{0}\right)$ the net of $f$ with respect to $v_{0}$.

One can describe all objects which can occur in this way. Let $\Gamma$ be a cell decomposition of $\mathbf{P}^{1}$ with the following properties:

(i) closure of each cell is homeomorphic to a closed ball of corresponding dimension;

(ii) $\Gamma$ is symmetric with respect to $\mathbf{T}$, that is the reflection $\mathbf{s}$ maps each cell of $\Gamma$ onto a cell of $\Gamma$;

(iii) all 0-cells belong to the unit circle $\mathbf{T}$ and there are $2 d-2$ of them;

(iv) 1-skeleton of $\Gamma$ contains $\mathbf{T}$.

As usual we call 0-, 1- and 2-cells vertices, edges and faces. The edges that do not belong to $\mathbf{T}$ will be called interior edges. A net is a pair $\gamma=\left(\Gamma, v_{0}\right)$ where $\Gamma$ is a cell decomposition of $\mathbf{P}^{1}$ with the properties (i)-(iv) and $v_{0}$ is a vertex of $\Gamma$.

Two nets $\left(\Gamma, v_{0}\right)$ and $\left(\Gamma^{\prime}, v_{0}^{\prime}\right)$ are called equivalent if there exists a homeomorphism $\phi$ of $\mathbf{P}^{1}$ preserving the orientations of both $\mathbf{P}^{1}$ and $\mathbf{T}$, commuting with the reflection $\mathbf{s}$ and having the properties $\phi(\Gamma)=\Gamma^{\prime}$ and $\phi\left(v_{0}\right)=v_{0}^{\prime}$. 
One of the results of [1] is the following: Let $f_{1}$ and $f_{2}$ be two rational functions in $R$ with the same critical points, and $v_{0}$ one of these common critical points. If the nets of $f_{1}$ and $f_{2}$ with respect to $v_{0}$ are equivalent, then $f_{1}$ and $f_{2}$ are equivalent.

The main technical result of [1] can be stated as follows: For every net $\gamma$ whose vertex set is $V$, and for every injective map $c: V \rightarrow \mathbf{T}$ preserving the cyclic order induced by the orientation, there exists a unique class $C$ of rational functions in $R$ whose critical points are $c(V)$ and whose net with respect to $c\left(v_{0}\right)$ is equivalent to $\gamma$.

We will say somewhat informally that functions of this class $C$ have prescribed net and prescribed critical points.

Functions $f \in R$ with a prescribed net and prescribed critical points can be normalized in the following way. Choose two additional vertices $v_{1}$ and $v_{-1}$ and three distinct points $w_{-1}, w_{0}, w_{1}$ on $\mathbf{T}$. Then the normalization condition is

$$
f\left(c\left(v_{i}\right)\right)=w_{i}, \quad i=-1,0,1 .
$$

There exists a unique normalized function $f \in R$ with a prescribed net and prescribed critical points. Another result from [1] says that for each fixed net, this normalized function depends continuously on the critical points, or more precisely, on the map $c$ above.

Now we can prove Theorem $1^{\prime}$. Suppose that the separated sets $A_{j} \subset \mathbf{T}$ satisfying (11) are given. Let $I_{j} \subset \mathbf{T}$ be disjoint arcs containing $A_{j}$. We list each $A_{j}$ as $\left(z_{j, 0}, z_{j, 1}, \ldots, z_{j, a_{j}}\right)$ where the order within $A_{j}$ is consistent with the counterclockwise orientation of $\mathbf{T}$. For any two distinct points $r$ and $s$ in $\mathbf{T}$, we denote by $[r, s]$ the closed arc of $\mathbf{T}$ which is traced from $r$ to $s$ counter-clockwise.

Choose additional points

$$
x_{j, k} \in\left[z_{j, k}, z_{j, k+1}\right], \quad 0 \leq k \leq a_{j}-1, \quad 1 \leq j \leq q .
$$

According to (11), the total number of these points $x_{j, k}$ is $2 d-2$. So we can use these points as vertices of a net. Let us use

$$
v_{0}=x_{1,0}
$$

in the definition of this net. This choice is arbitrary, any other point among $x_{j, k}$ can be taken as $v_{0}$.

We consider the nets satisfying the following additional condition:

$$
\text { there are no interior edges connecting two vertices on the same } I_{j} \text {. }
$$

Lemma 1. Suppose that a net $\gamma$ satisfies (19). Let all $x_{j, k}$, except one, $x=x_{j_{0}, k_{0}}$ be fixed arbitrarily, so that conditions (18) are satisfied. For fixed $w_{i} \in \mathbf{T}, j=0, \pm 1$, let $f_{x}$ be the function of the class $R$ with the net $\gamma$ and critical points $x_{j, k}$, normalized as in (17). Denote $I=\left[z_{j_{0}, k_{0}}, z_{j_{0}, k_{0}+1}\right]$. Then there exist a continuous branch of $\arg f_{x}(z)$, for $(x, z) \in I \times I$, such that the function

$$
\psi(x)=\arg f_{x}\left(z_{j_{0}, k_{0}}\right)-\arg f_{x}\left(z_{j_{0}, k_{0}+1}\right)
$$

changes sign as $x$ runs over $I$.

Proof. To simplify our notation we put $r=z_{j_{0}, k_{0}}, s=z_{j_{0}, k_{0}+1}$, so that $I=[r, s]$. Let $\ell$ be the interior edge of $\gamma$ that lies in the unit disc and has $x$ as an endpoint. Let $v$ be another endpoint of this edge. We note that neither the assumptions nor the conclusions of the lemma depend on the choice of the normalization. So we may assume that $v$ is one of the distinguished vertices, say $v=v_{1}$ in (17) and choose 
$w_{1}=f_{x}(v)=-1$. Let $D_{1}$ and $D_{2}$ be two faces of the net $\gamma$ in the unit disc which have $\ell$ as the common part of their boundaries. As the boundary of every face is mapped by $f_{x}$ bijectively onto $\mathbf{T}$, and the vertex $v$ is disjoint from $I$ because of (19) we conclude that $f_{x}(z) \neq-1$ for $(x, z) \in I \times I$. Thus $\arg f_{x}(z)$ can be defined as a continuous function for $(x, z) \in I \times I$, with values in $(-\pi, \pi)$. Now, $z \mapsto \arg f_{x}(z)$ is strictly monotone in the opposite directions on the arcs $[r, x]$ and $[x, s]$ : it is strictly increasing on one of these arcs and strictly decreasing on another one. This follows from the fact that one of the closed faces $D_{1}$ or $D_{2}$ is mapped homeomorphically onto the closed unit disc and another onto the closed exterior of the unit disc, while the arcs $[r, x]$ and $[x, s]$ are parts of the boundaries of $D_{1}$ and $D_{2}$.

We conclude that $\arg f_{x}(r)-\arg f_{x}(s)$ changes sign as $x$ runs from $r$ to $s$, which proves the lemma.

Lemma 2. If $\gamma$ is a net satisfying (19), then there exists a choice of critical points $x_{j, k}$ such that every function $f \in R$ with this net and with these critical points satisfies

$$
f\left(z_{j, 0}\right)=\ldots=f\left(z_{j, a_{j}}\right) \quad \text { for every } \quad j \in[1, q] .
$$

Proof. Let $\mathbf{x}$ be the vector with coordinates $\left(x_{j, k}\right)$. The set of $\mathbf{x}$ satisfying (18) is a closed cube $Q$. According to Lemma 1, the continuous functions $\psi_{j, k}(\mathbf{x})=$ $\arg f_{\mathbf{x}}\left(z_{j, k}\right)-\arg f_{\mathbf{x}}\left(z_{j, k+1}\right)$ with appropriate choices of branches of arguments take values of opposite signs on the opposite facets of $Q$. It follows from a corollary of Brouwer's Theorem [4, Ch. IV, 1D] that all these functions $\psi_{j, k}$ have a common zero in the interior of $Q$.

So, for each net $\gamma$ satisfying (19), Lemma 2 gives at least one function $f \in R$ that satisfies (20). Functions with different nets cannot be equivalent. The crucial fact is

Lemma 3. For each choice of the separated sets $A_{j}=\left\{z_{j, k}\right\}$ with card $A_{j}=a_{j}+1$ and vertices $x_{j, k}$ as in (18) there are exactly $K_{\boldsymbol{a}}$ nets with vertices $\left\{x_{j, k}\right\}$ and $v_{0}=x_{1,0}$, satisfying (19).

Proof. We describe two injective maps: one from the set of nets to the set of SSYT of shape $2 \times(n-1)$ (part A below) and another one in the opposite direction (part B).

We consider the real line instead of the unit circle, and suppose that $v_{0}=$ $x_{1,0}$ is the leftmost vertex. We also assume for convenience that the intervals are enumerated in such a way that $i<j$ implies $x<y$ for all $x \in I_{i}$ and $y \in I_{j}$, and the vertices in each interval are enumerated from left to right.

Part A. We construct an SSYT corresponding to a given net. To each vertex of the net we assign one entry in SSYT. These entries are defined inductively: for $j \geq 1$ and $n \geq 0$, suppose that the entries corresponding to $x_{i, k}$ for $i<j$, or $i=j$ and $k<n$ are already placed in the tableau. The entry corresponding to $x_{j, n}$ is equal to $j$. It is placed in the first row if the edge from $x_{j, n}$ goes forward (to some $x_{j^{\prime}, n^{\prime}}>x_{j, n}$ ) and in the second row if the edge from $x_{j, n}$ goes backward. The new entry $j$ is placed to the leftmost place in its row. It is easy to see that in this way we obtain an SSYT for each net satisfying (19).

Part $B$. In the opposite direction, we construct inductively the net corresponding to a given SSYT of shape $2 \times(d-1)$. On the $n$-th step of the construction we deal 
with a tableau of shape $2 \times(d-n)$ filled by positive integers such that the rows are non-decreasing and columns are strictly increasing. The only difference from SSYT is that the integers in the tableau are not necessarily consecutive; slightly abusing terminology we will call these tableaux SSYT as well. Assuming that such an SSYT is given, let $k$ be the first (leftmost) entry in its second row. We find the rightmost entry $m$ in the first row that is strictly less than $k$ and draw an edge between the leftmost free vertex in $I_{k}$ and the rightmost free vertex of $I_{m}$. Then we delete the first entry from the second row and the above defined entry from the first row, and shift the left part of the first row to the right to form a tableau of shape $2 \times(d-n-1)$. Evidently, the obtained tableau is an SSYT, so we can proceed until it becomes empty.

To complete Part B, it remains to prove that the edge added on the $n$-th step does not intersect the edges added on the previous steps.

Let us show first that on each step all the vertices lying between the endpoints of the added edge are not free. Indeed, suppose that there is a free vertex $v$ between the endpoints. We use the notation from the description of the $n$-th step above. By assumption, the vertex $v$ belongs to some $I_{p}$ with $m<p<k$, since the new edge joins the leftmost free vertex in $I_{k}$ to the rightmost free vertex in $I_{m}$. Since the vertex $v$ in $I_{p}$ is free there should be an entry $p$ somewhere in the current SSYT. It cannot be in the second row since $k>p$ is the leftmost entry of the second row. It cannot be in the first row since $m<p$ is the rightmost entry of those which are strictly less than $k$.

Now we can complete the proof. If a new edge intersects an old one, then one of the vertices of the new edge was a free vertex between the endpoints of the old edge on the step when this old edge was added. This completes the proof of the part B.

Now we can complete the proof of Theorem $1^{\prime}$. For given sets $A_{j}$ in general position, Schubert calculus gives at most $K_{\boldsymbol{a}}$ classes of rational functions solving Problem $1^{\prime}$. On the other hand, Lemmas 2 and 3 give at least $K_{\boldsymbol{a}}$ real classes of such functions. Thus all classes are real. Passing to the limit, we obtain that for all separated sets $A_{j}$, all solutions of Problem $1^{\prime}$ are real.

Proof of Theorem 3. Let $R^{\prime}$ be the subset of the closure of $R$ which consists of functions of degree exactly $d$. The nets of functions $f \in R^{\prime}$ have similar properties to those of $f \in R$, except that the vertex degrees can be arbitrary numbers between 4 and $2 d$. Such nets were called degenerate in 2 . In that paper, the following result was proved: Let $x_{1}^{a_{1}}, \ldots, x_{q}^{a_{q}}$ be a divisor on the real line, where $\boldsymbol{a}=\left(a_{1}, \ldots, a_{q}\right)$ satisfies (10) and let $\gamma$ be a net whose vertices enumerated in the increasing order have degrees $2 a_{j}+2$. Then there exists a real rational function of degree $d$ with critical points of multiplicity $a_{k}$ at the points $x_{k}$.

It is easy to see that the number of these degenerate nets $\gamma$ is the same as the number of non-degenerate nets satisfying (19), so according to Lemma 3, this number is $K_{\boldsymbol{a}}$. Hence the number of classes of real functions satisfying the conditions of Theorem 3 is at least $K_{\boldsymbol{a}}$ and this proves the theorem.

\section{REFERENCES}

[1] A. Eremenko and A. Gabrielov, Rational functions with real critical points and the B. and M. Shapiro conjecture in real enumerative geometry, Ann. Math., 155 (2002), 105-129. MR,1888795 (2003c:58028) 
[2] A. Eremenko and A. Gabrielov, Wronski map and Grassmannians of real codimension 2 subspaces, Computational Methods and Function Theory, 1 (2001) 1-25. MR 1931599 (2003h:26022)

[3] Ph. Griffiths and J. Harris, Principles of algebraic geometry, Willey-Interscience, NY, 1978. MR.0507725 (80b:14001)

[4] W. Hurewicz and H. Wallman, Dimension theory, Princeton Univ. Press, 1948. MR0006493 $(3,312 \mathrm{~b})$

[5] L. Manivel, Symmetric functions, Schubert Polynomials and degeneracy loci, AMS, Providence, RI, 2001. MR.1852463 (2002h:05161)

[6] J. Ruffo, Y. Sivan, E. Soprounova and F. Sottile, Experimentation and conjectures in the real Schubert calculus, work in progress, www.math.umass.edu/ sottile/pages/Flags.

[7] F. Sottile, The special Schubert calculus is real, Electron. Res. Announc. AMS, 5 (1999) 35-39. MR.1679451 (2000c:14074)

[8] F. Sottile, Real Schubert calculus: polynomial systems and a conjecture of Shapiro and Shapiro, Experimental Math., 9 (2000) 161-182. MR1780204(2001e:14054)

[9] F. Sottile, Enumerative real algebraic geometry, DIMACS Series in Discrete Math. and Computer Sci., vol. 60, AMS, Providence, RI (2003), 139-179. MR1995019 (2004j:14065)

[10] R. Stanley, Enumerative Combinatorics, vol. 2, Cambridge University Press, 1999. MR.1676282 (2000k:05026)

Department of Mathematics, Purdue University, West Lafayette, Indiana 47907-2067

E-mail address: eremenko@math.purdue.edu

Department of Mathematics, Purdue University, West Lafayette, Indiana 47907-2067

E-mail address: agabriel@math.purdue.edu

Department of Mathematics, Michigan State University, East Lansing, Michigan 48824

E-mail address: mshapiro@math.msu.edu

Department of Computer Science, University of Haifa, Mount Carmel, 31905 Haifa, ISRAEL

E-mail address: alek@cs.haifa.ac.il 